\title{
Rational Design and Synthesis of Novel Dual Protacs for Simultaneous Degradation of EGFR and PARP
}

Mengzhu Zheng, ${ }^{\#[a]}$ Junfeng Huo, ${ }^{\#[a]}$ Xiaoxia Gu, ${ }^{\#[a]}$ Canrong Wu, ${ }^{[a]}$ Qingzhe Zhang, ${ }^{[a]}$ Wang Wang ${ }^{[b]}$ Yang Liu, ${ }^{[b]}$ Yu Liu, ${ }^{[a]}$ Xuechen Zhou, ${ }^{[a]}$ Lixia Chen, ${ }^{*[b]}$ Yirong Zhou* ${ }^{*[a]}$ and Hua Li* ${ }^{[a, b]}$

a. Hubei Key Laboratory of Natural Medicinal Chemistry and Resource Evaluation, School of Pharmacy, Tongji-Rongcheng Center for Biomedicine, Tongji Medical College, Huazhong University of Science and Technology, Wuhan, 430030, China

b. Wuya College of Innovation, Key Laboratory of Structure-Based Drug Design \& Discovery, Ministry of Education, Shenyang Pharmaceutical University, Shenyang, 110016, China

* Corresponding authors:

syzyclx@163.com (L. Chen)

zhouyirong@hust.edu.cn (Y. Zhou)

li_hua@hust.edu.cn (H. Li)

\# M. Zheng, J. Huo, X. Gu contributed equally to this work.

Supporting information for this article is given via a link at the end of the document. 
Abstract: Inspired by the success of dual targeting drugs, especially bispecific antibodies, we propose to combine the concept of protac and dual targeting to design and synthesize dual protac molecules with the function of degrading two completely different types of targets simultaneously. A library of novel dual targeting protac molecules have been rationally designed and prepared. A convergent synthetic strategy has been utilized to achieve high synthetic efficiency. These dual protac structures are characterized by using trifunctional natural amino acids as star-type core linkers to connect two independent inhibitors and E3 ligands together. In this study, gefitinib, olaparib, and CRBN or VHL E3 ligand were used as substrates to synthesize novel dual protacs. They successfully degraded both EGFR and PARP simultaneously in cancer cells. Being the first successful example of dual protacs, this technique will greatly widen the range of application of the protac method and open up a new field for drug discovery.

In cancer, tumor cells often up-regulate different growth promoting factors, which can act independently or interfere with each other through a signal network. ${ }^{[1]}$ It is easy for tumor cells to acquire drug resistance by resorting to the compensatory factors or switching the signal pathway that promotes proliferation. ${ }^{[2]}$ Therefore, the treatment with drugs targeting only one single target inevitably exhibits limitations. In addition to drug resistance, the side effects and the tissue toxicity of single target drugs often lead to reduced efficacy and decreased life quality of patients. ${ }^{[3]}$ In order to overcome these shortcomings of single target drugs, drug combination targeting two or more different signal pathways of tumor has become a well-recognized effective method. ${ }^{[4]}$ Due to thesynergy effects of drug combination, a smaller dose of each single drug is needed, thus reducing the side effects.

Another approach to improve therapeutic efficacy is to design a single hybrid molecule merged by two or more pharmacophores to target two or more anti-tumor epitopes or targets simultaneously. ${ }^{[3]}$ Regulating multiple targets or pathways at the same time, these hybrid molecules usually exhibit better efficacy while causing fewer side effects. In past decades, these hybrid molecules, including bispecific antibodies and other small molecule drugs with double or multiple targets, ${ }^{[3,5]}$ have attracted great interest and achieved considerable success due to their superiority in the treatment of complex diseases. They have gradually become an alternative to combination therapy or the use of mixtures. ${ }^{[6,7]}$

Proteolysis targeting chimera (protac) is a kind of bifunctional small molecule, in which the target protein ligand and E3 ubiquitin ligase ligand are linked together through a linker to form a triplet compound. ${ }^{[8]}$ Compared with traditional small molecule inhibitors, protac has several advantages. It no more needs to bind to the active site of the target protein to exert effects, while it is capable of the degradation of "non-druggable" targets. There exists event driven mechanism rendering its 
catalytic properties, and it works at lower doses, while providing great opportunities for the development of anticancer drugs. ${ }^{[9]}$ However, the majority of reported protac molecules only connect one inhibitor with one E3 ligand, which often degrades only a single target protein and not exceeding the limit of two or more similar proteins.

Inspired by the great success of dual targeting drugs, especially bispecific antibodies, we envisage that by combining the concept of protac and dual targeting, a dual protac molecule with two independent inhibitor units and one E3 ligase ligand can be designed for degrading two targets simultaneously in completely different pathways (Figure 1). Dual protac can not only degrade two targets at the same time, but also imbibe merits of both protacs and double targeting drugs, achieving similar efficacy as bispecific antibodies, and still relatively conserving the advantages of small molecule drugs. To the best of our knowledge, there has been no report yet on this audacious and innovative idea. Therefore, it will be very interesting to design and synthesize such a novel dual protac molecule for proof of the concept.

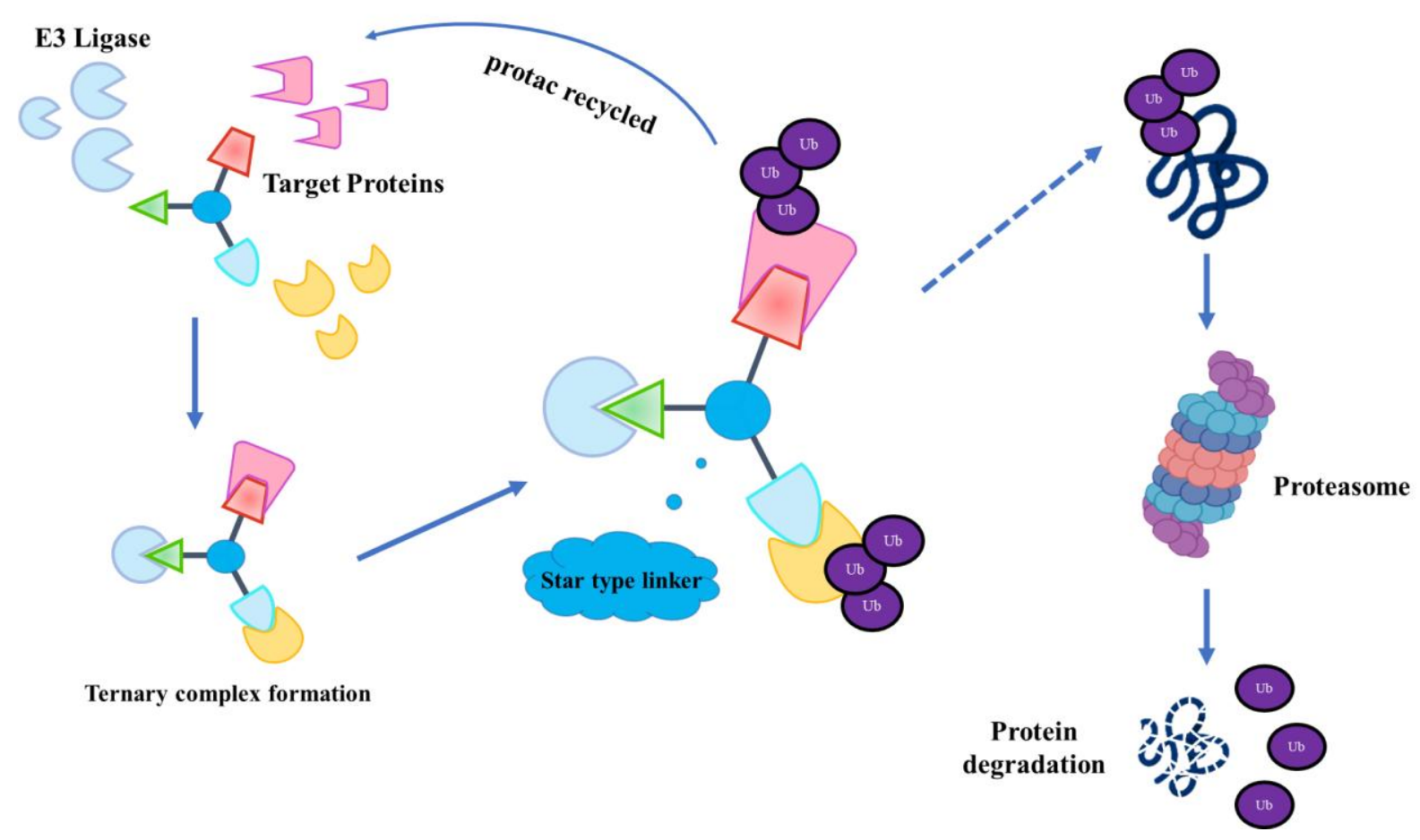

Figure 1. Novel star shape dual protacs and dual-targeting degradation. 

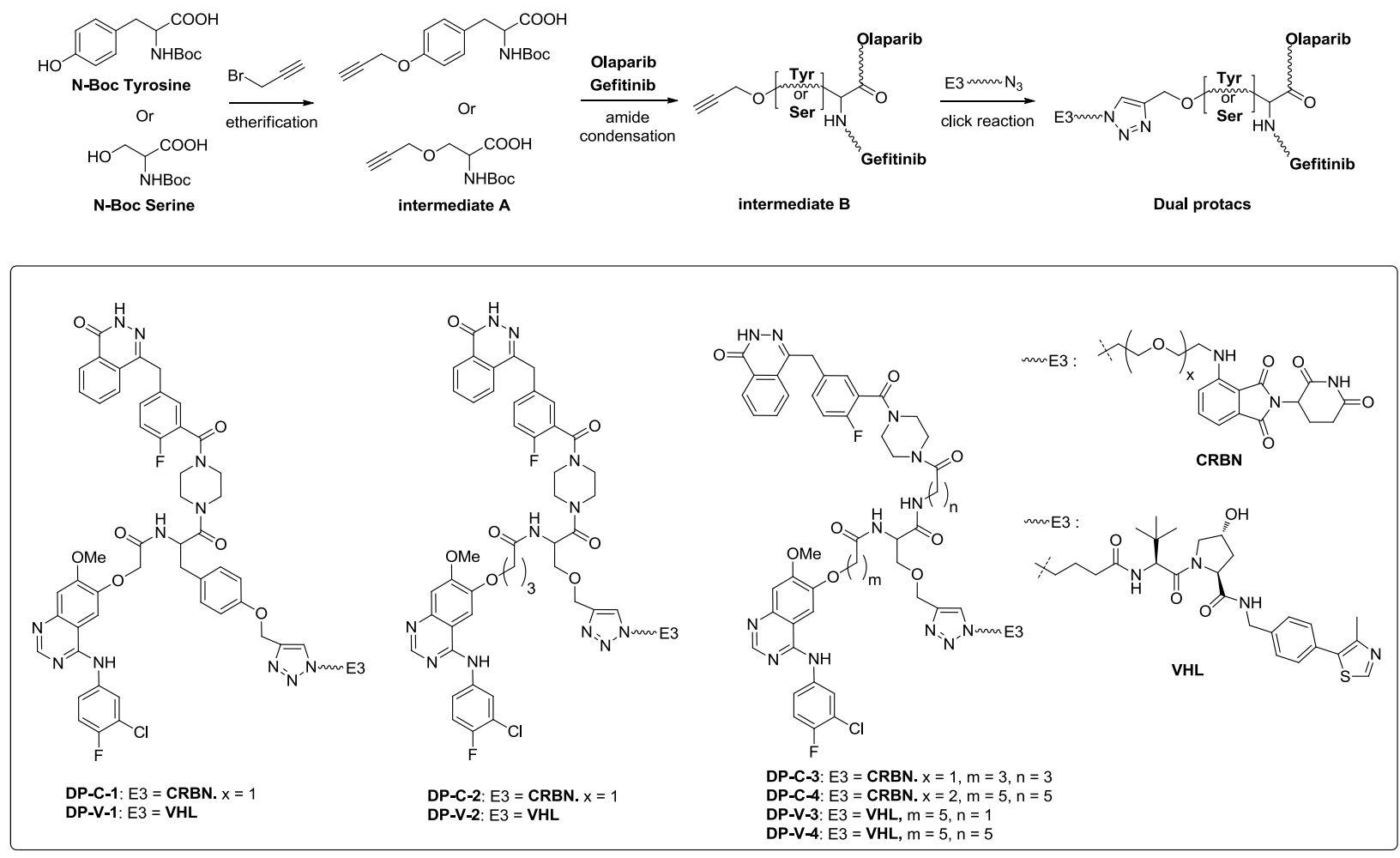

Scheme 1. Synthetic route for dual protacs and the structures of DP-C 1-4 and DP-V 1-4.

To verify our hypothesis shown above, the rational design of the novel star type linker should function as the most pivotal elements for dual protacs. Taking biocompatibility into consideration, natural amino acids will be a good choice. More importantly, some amino acids, such as tyrosine and serine, bearing a third reactive site besides normal amine and acid functional groups, are ideal star type linkers to connect three different small molecules (two inhibitors and one E3 ligand) together (Scheme 1). In addition, the three potential reactive sites display different reactivity, which makes them very suitable for sequential organic synthesis manipulation according to our rational design.

Drug resistance in advanced cancers has been reported to be mediated by different factors, such as epidermal growth factor receptor (EGFR) over-expression and DNA repair enzymes. Poly ADPribose polymerase (PARP) is one such protein that is known to be a key player in base excision repair $(B E R)^{[10]}$ and cellular signaling pathways. ${ }^{[11-12]}$ The inhibition of EGFR leads to the down-regulation of key players in BER and sensitizes cell response to alkylating agents and ionizing radiation. ${ }^{[13-14]}$ Receptor tyrosine kinase inhibitors (TKIs), just like EGFR inhibitors (e.g. Gefitinib), are widely used in clinic and have shown excellent therapeutic efficacy. ${ }^{[15-16]}$ However, the emergence of drug resistance, such as that due to T790M mutation, greatly reduces their efficacy. ${ }^{[17]}$ EGFR mutant cancer cells have been shown to be sensitive to Olaparib both in vivo and in vitro. ${ }^{[18]}$ Therefore, 
inhibition of both EGFR and PARP may produce a synergistic effect. ${ }^{[3]}$ In order to verify the concept of dual protac shown above, we designed the first round of dual protac molecules by merging EGFR inhibitors and PARP inhibitors with E3 ligase ligand in one novel star shape molecule, and evaluated their capability to degrade two independent targets at the cellular level.

As illustrated in Scheme 1, a convergent synthetic strategy was rationally designed based on our synthetic experience. The first etherification of the free hydroxyl group of tyrosine and serine with propargyl bromide provided the protected amino acid A. Subsequently, a sequential amide condensation reaction was utilized as a practical operation to induce Gefitinib and Olaparib to the star type linker, generating the key intermediate B. Eventually, the classical copper-promoted click reaction of azide and alkene was used to connect E3 ligands, CRBN and VHL, respectively. Based on this concise synthetic route, a small library of 8 dual protacs, including four CRBD ligand based protacs (DP-C-1-4, Figure S1), four VHL ligand based protacs (DP-V-1-4, Figure S2), and the corresponding four mono protacs in which one single inhibitor was linked (MP-GC, MP-GV, MP-OC, MP-OV, Figure S3) was efficiently constructed (For detailed synthetic procedures and structures, see the SI).

Due to drug resistance shown in pancreatic cancer and non-small cell lung cancer, as well as the high expression of EGFR and PARP, ${ }^{[19-24]}$ SW1990 and H1299 cell lines were used in the experiments. Intracellularly, the degradation of EGFR in the SW1990 cell line was induced by the CRBN based mono protac of Gefitinib (MP-GC) (Figure 2A). and the CRBN based mono protac of Olaparib (MP-OC) also exhibited a PARP degradation effect in the SW1900 cell line (Figure 2B). While neither Gefitinib or Olaparib itself could induce the degradation of two targets in SW1990 (Figure S4, S5).
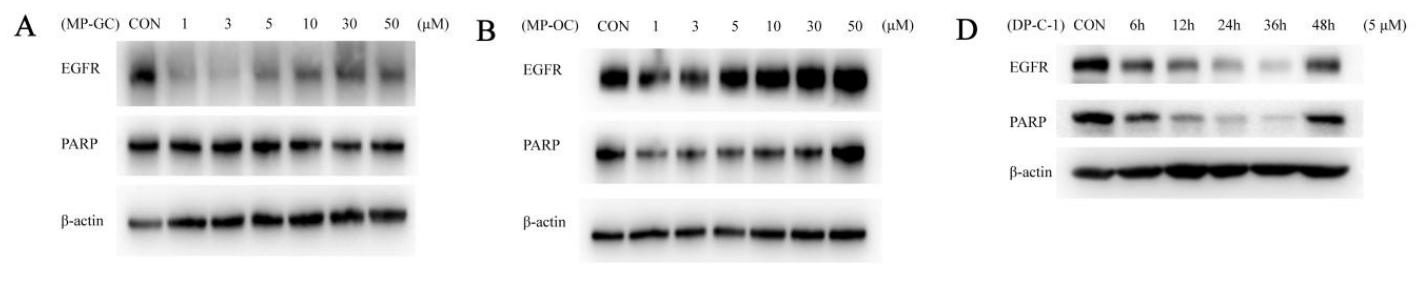

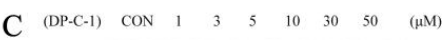

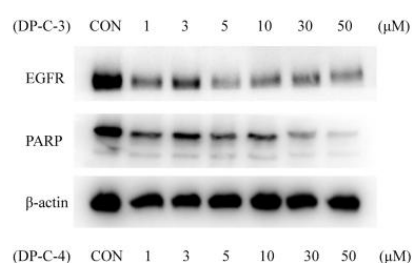

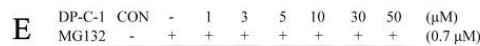
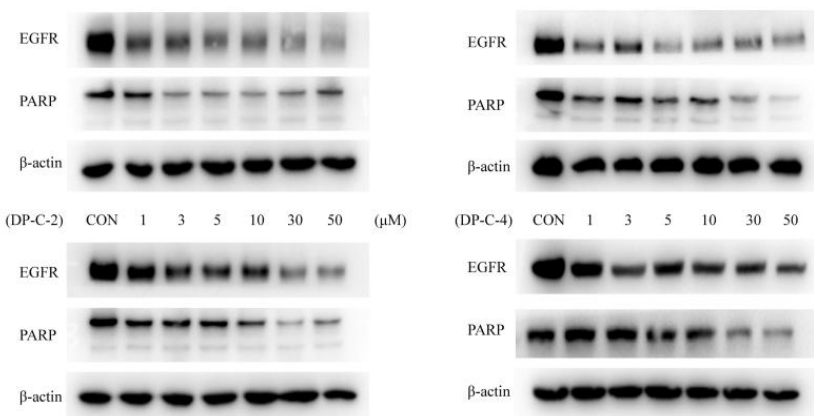

Figure 2. DP-C-1 degraded EGFR and PARP simultaneously in a dose- and time-dependent manner through the ubiquitin-proteasome system in SW1990 cells. A: Changes in associated proteins $24 \mathrm{~h}$ after addition of MP-GC. B: Changes in associated proteins $24 \mathrm{~h}$ after addition of MPOC. C: The effect of four dual protac compounds (DP-C 1-4) on the related proteins after $24 \mathrm{~h}$. D: The effect of $5 \mu M$ DP-C-1 on related proteins at different time points. E: Effect of DP-C-1 on related proteins after introduction of $700 \mathrm{nM} \mathrm{MG} 132$. 
Further experiments revealed that all four CRBN based dual protacs (DP-C-1-4) had degradation effects on EGFR and PARP simultaneously in SW1990 cells. As shown in Figure 2C, compound DP-C-1 showed the best degradation effect on both EGFR and PARP. The remaining compounds showed lower degradation ability on both targets compared to DP-C-1. These results also indicate that the degradation of EGFR and PARP by DP-C-1-4 was enhanced by increasing concentrations. The simultaneous degradation of EGFR and PARP by single dual protac molecule successfully proves the concept of dual targeting degradation.

To further demonstrate the degradation kinetics of DP-C-1, time-dependent experiments were carried out. Results showed that DP-C-1 began to degrade EGFR and PARP at $6 \mathrm{~h}$. At first, degradation rate increased with time going, but it started to decrease at $48 \mathrm{~h}$ (Figure 2D). Therefore, DP-C-1 degrades EGFR and PARP in SW1990 cell line in a time-dependent manner within $48 \mathrm{~h}$ and the optimal degradation rate was around 24 and $36 \mathrm{~h}$.

Protacs exert their degradation effect on target proteins through the ubiquitin-proteasome system. Therefore, MG132, a proteasome inhibitor, is widely used as a tool to verify the mechanism of such an action. ${ }^{[25-27]}$ To further verify that the degradation of proteins by DP-C-1 functions via the ubiquitination pathway, the effect of DP-C-1 was assayed by introducing the proteasome inhibitor MG 132. It can be seen from Figure 2E that due to the inhibitory effect of MG 132 itself on the SW1990 cells, the group with MG132 alone had a slightly lower expression level of both EGFR and PARP compared to that of the negative control (NC) group. However, the addition of DP-C-1 in various concentrations ceases to further decrease the expression levels of both targets, and they maintain approximately the same level. This shows that the degradation of DP-C-1 is inhibited by MG132. These results suggest that the degradation of the target protein induced by DP-C-1 functions via the proteasome pathway.

To further verify the concept of dual protac, another E3 ligase ligand (VHL-L) was chosen to develop new dual protacs. First, the corresponding mono protacs, MP-GV and MP-OV, were synthesized based on Gefitinib, Olaparib, and VHL ligand. In the H1299 cell line, MP-GV and MP-OV could induce EGFR and PARP degradation respectively, while exhibiting no effect on the other target protein (Figure 3A, 3B). According to this result, four dual protacs based on VHL ligand were further developed and designated as DP-V 1-4, respectively. To evaluate their degradation ability of EGFR and PARP, H1299 cells were treated with these four compounds under six different concentrations for $36 \mathrm{~h}$. Among the different compounds, DP-V-4 exhibited the best degradation ability for both EGFR and PARP (Figure 3C, 3D), and was selected for further investigation. Meanwhile, further experiments revealed that DP-V-4 could also degrade EGFR and PARP in human epidermal carcinoma A431 cells simultaneously (Figure $\mathrm{S6}$ ). 
In H1299 cells, DP-V-4 degraded EGFR and PARP simultaneously in a dose-dependent manner (Figure 3D). At the concentration of $0.47 \mu \mathrm{M}, \mathrm{DP}-\mathrm{V}-4$ could induce about $50 \%$ of PARP degradation, and as concentration increased, it induced PARP degradation to a greater level. For EGFR, DP-V-4 also induced degradation at higher levels when the compound concentration gradually increased. Moreover, DP-V-4 could significantly induce EGFR and PARP degradation at $15 \mu \mathrm{M}$. Apart from EGFR and PARP, p-EGFR concentrations decreased visibly when treated with DP-V-4, which could be the result of its degradation by DP-V-4, or reduced supply from EGFR.

To further explore the mode of action, different time points were set in $\mathrm{H} 1299$ cells after treated with $4 \mu \mathrm{M}$ of DP-V-4. At $6 \mathrm{~h}, \mathrm{DP}-\mathrm{V}-4$ showed the best degradation effect, and EGFR and PARP recovered as time went on (Figure $3 \mathrm{E}$ ), which might be explained by the accumulation of newly synthesized proteins in cells.

To further verify that the degradation of proteins in cells by DP-V-4 functions via the ubiquitination pathway, the effect of DP-V-4 was assayed by introducing the proteasome inhibitor MG 132. In H1299 cells, when pre-treated with $1 \mu \mathrm{M}$ MG132 for $4 \mathrm{~h}$, the degradation of EGFR and PARP by DP-V-4 completely terminated (Figure 3F). These results proved that DP-V-4 degrades EGFR and PARP through the ubiquitin-proteasome system.

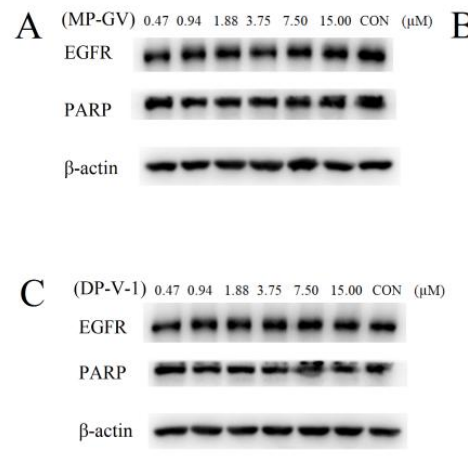

$\mathrm{E}$

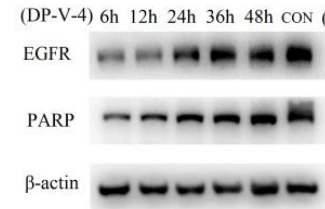

B
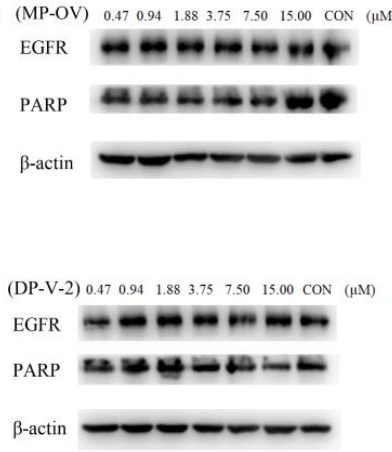

$\mathrm{F}$

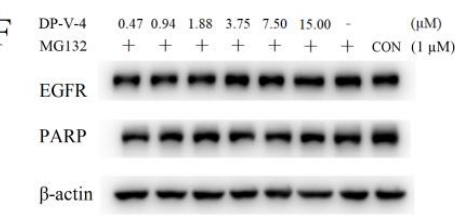

D (DP-V-4) $0.470 .94 \quad 1.883 .75 \quad 7.50 \quad 15.00 \quad \mathrm{CON} \quad(\mu \mathrm{M})$

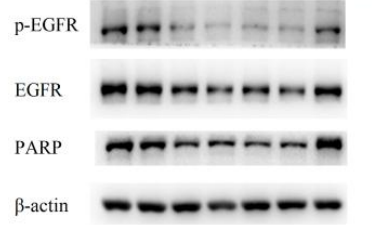

(DP-V-3) $0.470 .94 \quad 1.88 \quad 3.75 \quad 7.50 \quad 15.00$ CON $(\mu \mathrm{M})$

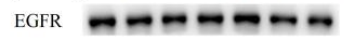

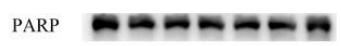

$\beta$-actin $=-\infty=-\infty$

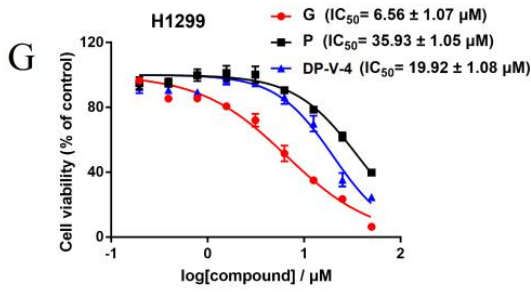

Figure 3. DP-V-4 degraded EGFR and PARP simultaneously in a dose- and time-dependent manner through the ubiquitin-proteasome system in H1299 cells. A: Changes in associated proteins $36 \mathrm{~h}$ after addition of MP-GV. B: Changes in associated proteins $36 \mathrm{~h}$ after addition of MP-OV. C: The effect of three dual protac compounds (DP-V 1-3) on related proteins after $36 \mathrm{~h}$. D: The effect of DP-V-4 on related proteins with different concentration at $36 \mathrm{~h}$. E: The effect of $4 \mu \mathrm{M}$ DP-V-4 on related proteins at different times. F: Effect of DP-V-4 on related proteins after introduction of $1 \mu \mathrm{M}$ MG132. G: The anti-proliferative activity of Gefitinib, Olaparib and DP-V-4 was identified by CCK8 assay. IC50 was expressed as mean \pm SD. 
Besides degradation ability, we attempted to identify the anti-proliferative activity of dual protacs in H1299 tumor cells. CCK8 assay determined Gefitinib, Olaparib and DP-V-4 with IC50s of $6.56 \pm$ $1.07 \mu \mathrm{M}, 35.93 \pm 1.05 \mu \mathrm{M}$ and $19.92 \pm 1.08 \mu \mathrm{M}$, respectively (Figure 3G). The relatively weaker antiproliferative activity of DP-V-4 might be explained by its poorer solubility and cell penetrability, owing to its higher molecular weight.

A

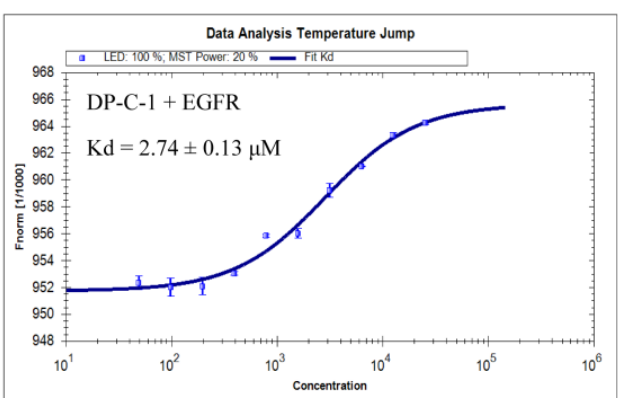

B

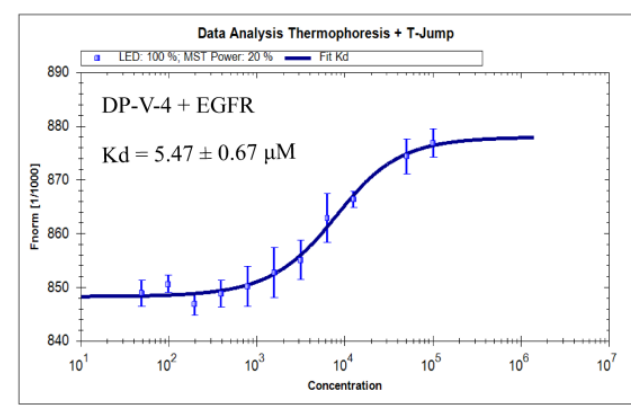

$\mathrm{C}$

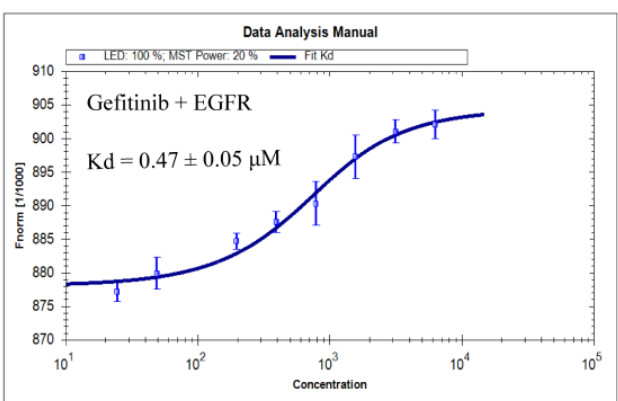

$\mathrm{D}$

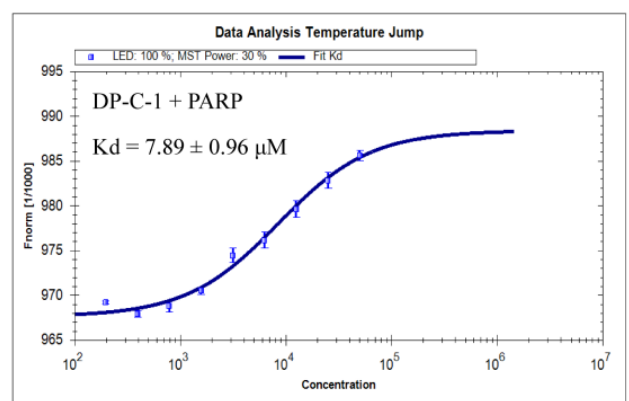

E

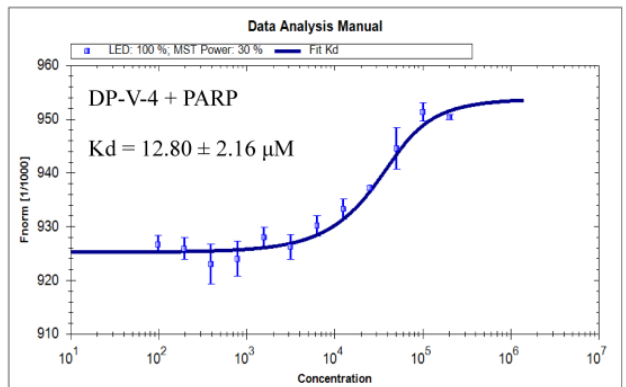

$\mathrm{F}$

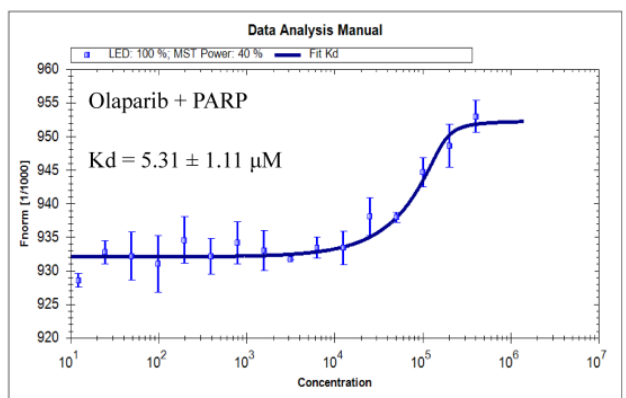

Figure 4. The binding affinity of DP-C-1 and DP-V-4 with EGFR and PARP. A-C: Measurement of affinity of DP-C-1, DP-V-4, and Gefitinib to EGFR by MST with Kd values of $2.74 \pm 0.13,5.47 \pm 0.67$, and $0.47 \pm 0.05 \mu \mathrm{M}$, respectively. D-F: Measurement of affinity of DP-C-1, DP-V-4, and Olaparib to PARP by MST with Kd values of $7.89 \pm 0.96,12.80 \pm 2.16$, and $5.31 \pm 1.11 \mu \mathrm{M}$, respectively.

In order to further confirm the target engagement of these dual protacs, MST was employed to assay the binding affinity between the two targets and two representative dual protacs, DP-C-1 (CRBN based) and DP-V-4 (VHL based). As shown in Figure 4, although a little weaker binding was observed for both DP-C-1 and DP-V-4 compared to Gefitinib with EGFR (Kd $0.47 \pm 0.05 \mu \mathrm{M}$ ) and Olaparib with PARP (Kd $5.31 \pm 1.11 \mu \mathrm{M}$ ), they still kept strong binding with EGFR and PARP, with the disassociation constants for DP-C-1 at $2.74 \pm 0.13 \mu \mathrm{M}$ and $7.89 \pm 0.96 \mu \mathrm{M}$ with EGFR and PARP, 
respectively, and for DP-V-4 at $5.47 \pm 0.67 \mu \mathrm{M}$ and $12.80 \pm 2.16 \mu \mathrm{M}$ with EGFR and PARP, respectively.

In this study, we proposed the new concept of dual targeting protac. Inspired by bispecific antibodies, $^{[5]}$ we creatively proposed the idea of designing a dual targeting protac molecule with two different warheads, in which an E3 ligand was linked to two inhibitors with different types/pathways of targets, and was capable of simultaneously degrading two completely different target proteins in tumor cells. In order to verify this concept, we used Gefitinib (an existing EGFR inhibitor) and olaparib (a PARP inhibitor) ${ }^{[3]}$ as substrates to synthesize dual targeting degradation chimeric molecules (dual protac) of EGFR and PARP proteins with different linker length and different E3 ligand (CRBN or VHL), which successfully degraded EGFR and PARP simultaneously in cancer cells. This is the first successful example of dual protacs.

In addition, we used biocompatible natural amino acids as a linker to achieve the rapid synthesis of star type dual targeting protacs. The technique and method established in this study can be easily adapted for the synthesis of more dual targeting protac molecules just like building blocks, and it will greatly expand the application of protac technology while opening up a new avenue for drug discovery. In present clinical practice, the combination therapy of two different inhibitors, or even the treatment of some bispecific antibodies, could be substituted by the strategy shown in this study through designing a corresponding single dual protac molecule for double targeting degradation, with the same or even better therapeutic effects.

It is easy to recognize that in the field of combination therapy of cancer, dual protacs can be quickly applied to replace many current combinations. For example, we could design and synthesize the dual protacs targeting two kinases which are synthetically lethal, or targeting a tumor immune target plus an adjuvant immune target, kinase, or energy metabolism target, or targeting an epigenetic target plus an anti-apoptotic target, etc. Of course, in addition to the obvious advantages listed above, the increased molecular weight of dual protacs will give rise to some issues in the aspect of drug properties and pharmacokinetics. The approach to solving these problems lies in two directions. One is to employ a nanodrug delivery system to improve drug absorption, and the other is to simplify the inhibitor part while retaining the minimum pharmacophore. We are currently working on realizing these approaches.

In conclusion, we have designed and synthesized the first dual targeting protac molecules with two different warheads, and realized the simultaneous degradation of two completely different types of target proteins by one small molecule in tumor cells. We believe that this technique will greatly expand the application of protac technology and open up a new field of drug discovery. 


\section{Acknowledgements}

This work was supported by the National Natural Science Foundation of China (NSFC) (grant numbers 81773637, U1803122, and 81903863), National Mega-project for Innovative Drugs (grant number 2019ZX09721001-004-007, China), Chunhui Program-Cooperative Research Project of the Ministry of Education, the Natural Science Foundation of Hubei Province (No. 2020CFB642), 100 Talents Program of the Hubei Provincial Government, Liaoning Revitalization Talents Program (No. XLYC1807182), Liaoning Province Natural Science Foundation (No. 2020-MZLH-31).

Keywords: dual protac $\bullet$ EGFR $\bullet$ PARP • protein degradation • star type linker

\section{References}

[1] J. van der Veeken, S. Oliveira, R. M. Schiffelers, G. Storm, P. M. van Bergen En Henegouwen, R. C. Roovers, Curr. Cancer Drug Tar. 2009, 9,748-60.

[2] F. Morgillo, H. Y. Lee, Drug Resist. Update. 2005, 8, 298-310.

[3] A. Anighoro, J. Bajorath, G. Rastelli, J. Med. Chem. 2014, 57, 7874-7887.

[4] J. J. Wright, Cli. Cancer Res. 2010, 16, 4094-4104.

[5] R. E. Kontermann, mAbs 2012, 4, 182-197.

[6] J. Schmitt, S. Huang, E. Goodfellow, C. Williams, B. J. Jean-Claude, J. Med. Chem. 2020, 63, 5752-5762.

[7] T. Di Desidero, A. Fioravanti, P. Orlandi, B. Canu, R. Giannini, N. Borrelli, S. Man, P. Xu, G. Fontanini, F. Basolo, R. S. Kerbel, G. Francia, R. Danesi, G. Bocci, J. Clin. Endocrinol. Metab. 2013, 98, E1465-1473.

[8] A. C. Lai, C. M. Crews, Nat. Rev. Drug. Discov. 2017, 16, 101-114.

[9] M. Schapira, M. F. Calabrese, A. N. Bullock, C. M. Crews, Nat. Rev. Drug. Discov. 2019, 18, 949-963.

[10] A. R. Chaudhuri, A. Nussenzweig, Nat. Rev. Mol. Cell Biol. 2017, 18, 610-621.

[11] M. S. Cohen, P. Chang, Nat. Chem. Biol. 2018, 14, 236-243.

[12] R. Smith, T. Lebeaupin, S. Juhasz, C. Chapuis, O. D'Augustin, S. Dutertre, P. Burkovics, C. Biertumpfel, G. Timinszky, S. Huet, Nucleic Acids Res. 2019, 47, 11250-11267.

[13] A. Yacoub, R. McKinstry, D. Hinman, T. Chung, P. Dent, M. P. Hagan, Radiat. Res. 2003, 159, 439-452.

[14] J. Schmitt, S. Huang, E. Goodfellow, C. Williams, B. J. Jean-Claude. J. Med. Chem. 2020, 63, 57525762

[15] W. Pao, J. Chmielecki, Nat. Rev. Cancer 2010, 10, 760-74.

[16] J. G. Paez, P. A. Janne, J. C. Lee, S. Tracy, H. Greulich, S. Gabriel, P. Herman, F. J. Kaye, N. Lindeman, T. J. Boggon, K. Naoki, H. Sasaki, Y. Fujii, M. J. Eck, W. R. Sellers, B. E. Johnson, M. Meyerson, Science 2004, 304, 1497-500. 
[17] C. K. Lee, L. Davies, Y. L. Wu, T. Mitsudomi, A. Inoue, R. Rosell, C. Zhou, K. Nakagawa, S. Thongprasert, M. Fukuoka, S. Lord, I. Marschner, Y. K. Tu, R. J. Gralla, V. Gebski, T. Mok, J. C. Yang, J Natl. Cancer. Inst. 2017, 109, djw279.

[18] H. N. Pfaffle, M. Wang, L. Gheorghiu, N. Ferraiolo, P. Greninger, K. Borgmann, J. Settleman, C. H. Benes, L. V. Sequist, L. Zou, H. Willers, Cancer Res. 2013, 73, 6254-63.

[19] F. R. Hirsch, G. V. Scagliotti, J. L. Mulshine, R. Kwon, W. J. Curran, Y.-L. Wu, L. Paz-Ares, Lancet 2017, 389, 299-311.

[20] R. S. Herbst, M. Fukuoka, J. Baselga, Nat. Rev. Cancer 2004, 4, 956-965.

[21] F. Cappuzzo, T. Ciuleanu, L. Stelmakh, S. Cicenas, A. Szczesna,'E. Juhasz, E. Esteban, O. Molinier, W. Brugger, I. Melezínek,' G. Klingelschmitt, B. Klughammer, G. Giaccone, Lancet Oncol. 2010, 11, 521529.

[22] M. D. Muzumdar, P. Y. Chen, K. J. Dorans, K. M. Chung, A. Bhutkar, E. Hong, E. M. Noll, M. R. Sprick, A. Trumpp, T. Jacks, Nat. Commun. 2017, 8, 1090.

[23] S. Shankar, J. C. Tien, R. F. Siebenaler, S. Chugh, V. L. Dommeti, S. Zelenka-Wang, X. M. Wang, I. J. Apel, J. Waninger, S. Eyunni, A. Xu, M. Mody, A. Goodrum, Y. Zhang, J. J. Tesmer, R. Mannan, X. Cao, P. Vats, S. Pitchiaya, S. J. Ellison, J. Shi, C. Kumar-Sinha, H. C. Crawford, A. M. Chinnaiyan. Nat. Commun. 2020, 11, 2817.

[24] C. Du, Y. Qi, Y. Zhang, Y. Wang, X. Zhao, H. Min, X. Han, J. Lang, H. Qin, Q. Shi, Z. Zhang, X. Tian, G. J. Anderson, Y. Zhao, G. Nie, Y. Yang, ACS Nano. 2018, 12, 10785-10796.

[25] K. He, Z. Zhang, W. Wang, X. Zheng, X. Wang, X. Zhang, Bio. Med. Chem. Lett. 2020, 30, 127167.

[26] H. Zhang, H. Y. Zhao, X. X. Xi, Y. J. Liu, M. Xin, S. Mao, J. J. Zhang, A. X. Lu, S. Q. Zhang, Euro. J. Med. Chem. 2020, 189, 112061.

[27] X. Zhang, F. Xu, L. Tong, T. Zhang, H. Xie, X. Lu, X. Ren, K. Ding, Euro. J. Med. Chem. 2020, 192, 112199. 